\title{
Severe esophageal bleeding in colorectal cancer due to antitumor therapy: A case report
}

\author{
HONG SHEN ${ }^{1}$, XIAN-YUN YE ${ }^{1}$, XIAO-FEN LI ${ }^{1}$, WEN-SHENG PAN $^{2}$ and YING YUAN ${ }^{1}$ \\ Departments of ${ }^{1}$ Medical Oncology and ${ }^{2}$ Gastroenterology, \\ Second Hospital of Zhejiang University College of Medicine, Hangzhou, Zhejiang 310009, P.R. China
}

Received September 3, 2014; Accepted May 20, 2015

DOI: $10.3892 / \mathrm{ol} .2015 .3742$

\begin{abstract}
Colorectal cancer is the third most common type of cancer worldwide, with $>1$ million cases diagnosed each year. Gastrointestinal bleeding is a common complication of colorectal cancer and is usually associated with the erosion and hemorrhage of the primary tumor. However, in patients who undergo a radical hemicolectomy and do not develop local recurrence, gastrointestinal bleeding may be a result of medical treatments or comorbidities. Esophageal bleeding in such patients is rare. Here, a case of severe esophageal bleeding due to anti-angiogenesis therapy with bevacizumab, and chemotherapy with the FOLFIRI regimen (irinotecan, folinic acid and 5-fluorouracil) in a patient with colorectal cancer is reported, and the possible pathogenesis of this event is analyzed based on the existing literature, in order to provide a reference for such cases.
\end{abstract}

\section{Introduction}

Acute gastrointestinal bleeding is a common medical emergency, which is most often a result of peptic ulceration, invasive esophageal inflammation, esophageal and gastric varices, and malignant tumors (1). Of colorectal cancers, $34.5 \%$ present with chronic melena, commonly associated with erosion and hemorrhage of the primary tumor (2). A number of cases of patients presenting with acute massive hemorrhage from the rectum have been reported (3-7). Acute massive hemorrhage from the upper gastrointestinal tract is rare in patients with colorectal cancer. Thorpe et al (8) reported one case of a patient with acute gastric bleeding caused by colorectal cancer involving the distal transverse colon, with a fistulous communication to the stomach. In patients in whom the primary colonic tumor has been resected, common reasons for bleeding

Correspondence to: Professor Ying Yuan, Department of Medical Oncology, Second Hospital of Zhejiang University College of Medicine, 88 Jie-fang Road, Hangzhou, Zhejiang 310009, P.R. China E-mail: yuanying1999@zju.edu.cn

Key words: colorectal cancer, esophageal bleeding, chemotherapy, bevacizumab include duodenal stress ulcers and anastomotic fistulae (9). The present report discusses a case of severe esophageal hemorrhage in a patient with colorectal cancer, with good surgical wound healing and no local recurrence, in whom anticancer treatment was proposed to be the primary cause of bleeding.

\section{Case report}

A 72-year-old Chinese male underwent a radical right hemicolectomy for colorectal cancer (pT4NOM0, Stage II) in a Xijing Hospital (Xi'an, China) in February 2011. The pathology report revealed an ulcerative adenocarcinoma in the ascending colon with infiltration to surrounding fatty tissues, without lymph node involvement and with a negative surgical margin. Genetic analysis indicated the presence of the K-ras mutation (G12D) and UGT1A1 6/7 type. The patient was considered as high risk stage II, and therefore received adjuvant chemotherapy with 6 cycles of FOLFOX4 and regular follow-up. Abdominal enhanced CT and PET-CT scans demonstrated multiple liver nodes in October 2011, two months after the administration of adjuvant chemotherapy. This confirmed the presence of unresectable liver metastases. Subsequently, anti-angiogenesis therapy with bevacizumab, and chemotherapy with the FOLFIRI regimen [irinotecan, $180 \mathrm{mg} / \mathrm{m}^{2}$ IV over $90 \mathrm{~min}$; folinic acid, $400 \mathrm{mg} / \mathrm{m}^{2} \mathrm{IV}$ over $2 \mathrm{~h}$; 5-fluorouracil (5-FU), bolus of $400 \mathrm{mg} / \mathrm{m}^{2} \mathrm{IV}$; 5-FU, continuous infusion of $2400 \mathrm{mg} / \mathrm{m}^{2} 46 \mathrm{~h}$ ] and bevacizumab (5 mg/kg IV over $90 \mathrm{~min}$ ) were administered in Xijing Hospital. On day 2, the patient presented with severe diarrhea, which abated following treatment with loperamide. The following day, the patient developed abdominal pain, abdominal bloating and vomiting, and was unable to defecate. An abdominal X-ray demonstrated intestinal obstruction. Supportive therapy was administered until the symptoms had resolved. On day 7 , the patient's blood count indicated severe bone marrow toxicity of grade IV, with white blood cells of $0.9 \times 10^{9} / 1$. Granulocyte-colony stimulating factor was administered for five days, and the patient's white blood cell count increased to $10 \times 10^{9} / 1$. On day 12, the patient vomited $600 \mathrm{ml}$ fresh blood, following a period of nausea, and also excreted $400 \mathrm{ml}$ black stools. The initial physical examination demonstrated weakness and pallor, with normal vital signs. The laboratory tests showed that the fecal occult blood test was positive (3+), the serum albumin was $28.2 \mathrm{~g} / \mathrm{l}$ and the initial hemoglobin was $10.1 \mathrm{~g} / \mathrm{dl}$. Therefore, the patient was treated with fluid replacement, octreotide and 
losec, and noradrenaline was infused through a nasogastric tube. When the patient had been stabilized, an endoscopy was conducted. This demonstrated diffuse ulceration and fistulae in the submucosa of the esophagus, old hemorrhage, mild hyperemia and scattered erosions in the mucosa of the gastric body, as well as in the gastric antrum and duodenum (Fig. 1). The patient had undergone an endoscopy in January 2011, during which no obvious abnormalities were detected. Furthermore, the colonoscopy did not show any evidence of tumor relapse or a bleeding lesion. The following day, the patient developed shock, with a blood pressure of $60 / 30 \mathrm{mmHg}$, a temperature of $38.9^{\circ} \mathrm{C}$, tachycardia at 126 beats/min and hemoglobin of $6.9 \mathrm{~g} / \mathrm{dl}$. Massive upper gastrointestinal bleeding was suspected. Following transfusion with two units of red blood cells, four units of plasma, and treatment with antibiotics and dopamine, the patient's condition improved.

Written informed consent for the present study was obtained from the patient.

\section{Discussion}

In the case reported, a patient who had undergone excision of the primary tumor and exhibited no evidence of local relapse, developed acute severe esophageal bleeding after one cycle of the FOLFIRI regimen and bevacizumab. Colonoscopy of the entire colorectal tract, demonstrated neither relapse nor a bleeding lesion. Gastroscopy showed diffuse ulceration and fistulae in the esophageal submucosa, which were presumed to be bleeding lesions. The occurrence of bleeding may have been a result of mucosal injury caused by chemotherapy, mucosal vascular dysfunction induced by bevacizumab or the combined effects of both. Yokoyama et al (10) reported that during continuous infusion chemotherapy with 5-FU, two patients presented with bloody stools secondary to enterocolitis. In a separate report, eight patients with hepatic cell carcinoma presented with massive bleeding caused by injury of the gastroduodenal artery, secondary to extravasation of 5-FU during hepatic arterial chemoperfusion (11). Chemotherapy with irinotecan may result in a colonic tumor bleeding directly or indirectly. The incidence of gastrointestinal bleeding from a primary tumor was $4 \%$, in a study examining the effect of the AIO regimen (weekly high-dose 5-FU as a 24-h infusion plus folinic acid) in patients with colorectal cancer (12). Yokoyama et al (10) also reported one patient who developed acute massive gastrointestinal bleeding on the 31st day after the first cycle of irinotecan-based chemotherapy, caused by a tumor of the transverse colon that had metastasized from lung cancer. Irinotecan is known to cause severe diarrhea, particularly in patients in whom the UGT1A1 gene is of the 7/7 type, which further induces bleeding, secondary to enterocolitis (10). Moriwaki et al (13) reported that the incidence of bleeding was higher in a group treated with irinotecan following an oxaliplatin regimen (6/115), compared with that in a group treated with oxaliplatin following an irinotecan regimen (0/45). Secondly, bevacizumab, a monoclonal antibody against the vascular endothelial growth factor receptor, increases the efficacy when given, in combination with chemotherapy, to patients with metastatic colorectal cancer. Anti-angiogenesis agents may lead to dysfunction of vascular endothelial cells and decrease their capacity for regeneration, which contributes to bleeding. In terms of adverse effects [categorized according

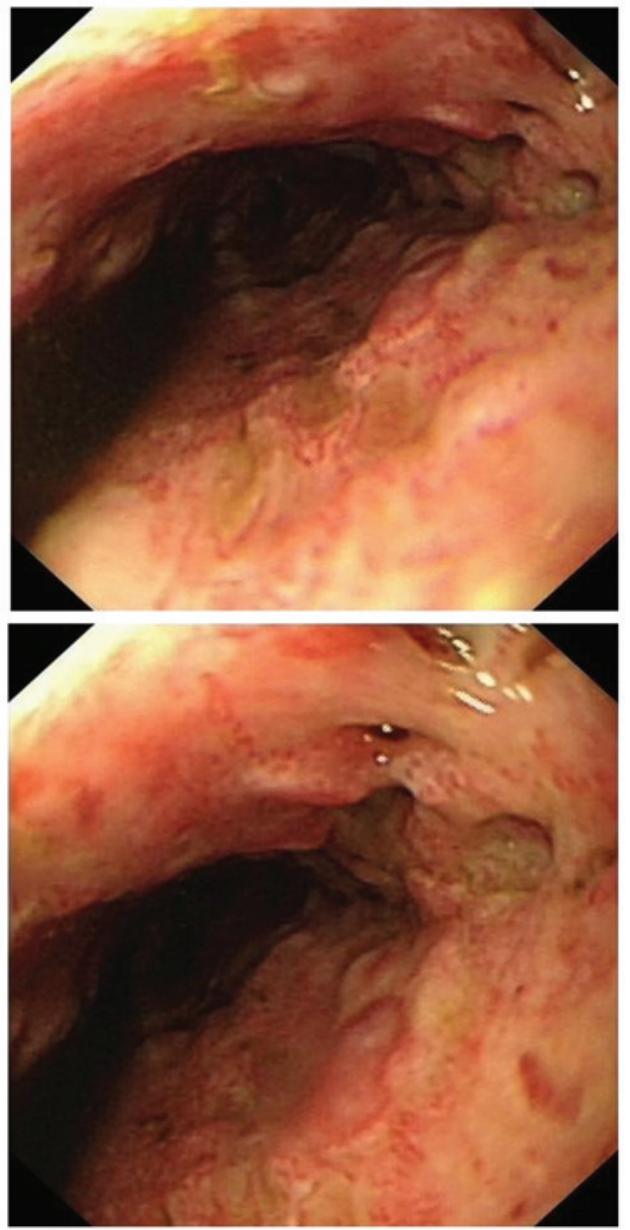

Figure 1. Endoscopy indicated diffuse ulceration and fistulae in the submucosa of the esophagus, in addition to remote hemorrhage.

to the Common Toxicity Criteria of the National Cancer Institute (14)], Hurwitz et al (15) reported that the incidence of grade 3 or 4 bleeding was higher in patients administered the irinotecan, bolus fluorouracil, and leucovorin (IFL) regimen plus bevacizumab (3.1\%) when compared with those receiving the IFL regimen without bevacizumab (2.5\%). Furthermore, Giantonio et al (16) reported that the incidence of grade 3 or 4 bleeding was higher in patients receiving the FOLFOX4 regimen plus bevacizumab (3.4\%) when compared with patients treated with the FOLFOX4 regimen alone $(0.4 \%)$ or bevacizumab alone (2.1\%). In addition, various bleeding locations were reported, including the primary tumor, articular cavities, the eyeball, and encephalic and perinephric spaces (17-19). The primary tumor was the most common bleeding site, accounting for $2.7 \%$ of cases. Tsuchida et al (20) reported a case of a patients with colorectal cancer, who presented with anal bleeding caused by shrinkage of an area of local relapse, following treatment with XELOX and bevacizumab.

Although there are numerous causes of upper gastrointestinal bleeding, the principles of treatment are similar. Once hemorrhage occurs, all potentially causative drugs should be stopped immediately. Vital signs should be closely monitored, and anticoagulant drugs, fluid infusion and blood transfusion administered as required. The timing of endoscopy should be assessed on an individual basis. There are risks associated with performing an emergency endoscopy. However, it carries 
diagnostic and therapeutic benefits when performed by experienced doctors. In the present case, when bleeding was confirmed, supportive therapy and endoscopy were instigated appropriately.

In conclusion, during the treatment of advanced colorectal cancer, particularly in patients treated with chemotherapy combined with anti-angiogenesis agents, it is important to remain aware of the possibility of gastrointestinal bleeding, to consider uncommon sources of such bleeding and to provide effective and timely management. Once bleeding is confirmed, intervention should be provided promptly. Even when severe bleeding occurs, satisfactory outcomes may be achieved with expeditious active treatment.

\section{Acknowledgements}

This study was funded by the National Natural Science Foundation of China (grant nos. 81102013 and 81201640).

\section{References}

1. El-Tawil AM: Trends on gastrointestinal bleeding and mortality: Where are we standing? World J Gastroenterol 18: 1154-1158, 2012

2. Courtney RJ, Paul CL, Sanson-Fisher RW, Macrae FA, Attia J and McEvoy M: Factors associated with consultation behaviour for primary symptoms potentially indicating colorectal cancer: A cross-sectional study on response to symptoms. BMC Gastroenterol 12: 100, 2012.

3. Chen CW, Jao SW, Wu CC, Ou JJ, Hsiao CW and Chao PC: Massive lower gastrointestinal hemorrhage caused by a large extraluminal leiomyoma of the colon: Report of a case. Dis Colon Rectum 51: 975-978, 2008.

4. Casarella WJ, Kanter IE and Seaman WB: Right-sided colonic diverticula as a cause of acute rectal hemorrhage. N Engl J Med 286: 450-453, 1972.

5. Leitman IM, Paull DE and Shires GT 3rd: Evaluation and management of massive lower gastrointestinal hemorrhage. Ann Surg 209: 175-180, 1989

6. Al Qahtani AR, Satin R, Stern J and Gordon PH: Investigative modalities for massive lower gastrointestinal bleeding. World J Surg 26: 620-625, 2002.

7. Imdahl A, Salm R, Rückauer K and Farthmann EH: Diagnosis and management of lower gastrointestinal hemorrhage. Retrospective analysis of 233 cases. Langenbecks Arch Chir 376: 152-157, 1991 (In German).

8. Thorpe B, Applebaum B, Esquivel RF, Krouse RS and Fass R: Colon cancer presenting as upper-GI bleeding. Gastrointest Endosc 63: 343-345, 2006
9. Gralnek IM: Gastrointestinal bleeding. Gastrointest Endosc 76: 506-509, 2012

10. Yokoyama T, Kondo H, Yokota T, Tokue Y, Saito D, Shimada Y and Sugihara K: Colonoscopy for frank bloody stools associated with cancer chemotherapy. Jpn J Clin Oncol 27: 111-114, 1997.

11. Ross WB, Morris DL and Clingan PR: Major upper gastrointestinal haemorrhage associated with hepatic arterial chemoperfusion. Aust NZ J Surg 66: 816-819, 1996.

12. Koucky K, Wein A, Konturek PC, Albrecht H, Reulbach U, Männlein G, Wolff K, Ostermeier N, Busse D, Golcher H, et al: Palliative first-line therapy with weekly high-dose 5-fluorouracil and sodium folinic acid as a 24-hour infusion (AIO regimen) combined with weekly irinotecan in patients with metastatic adenocarcinoma of the stomach or esophagogastric junction followed by secondary metastatic resection after downsizing. Med Sci Monit 17: CR248-CR258, 2011.

13. Moriwaki T, Bando H, Takashima A, Yamazaki K, Esaki T, Yamashita K, Fukunaga M, Miyake Y, Katsumata K, Kato S, et al: Bevacizumab in combination with irinotecan, 5-fluorouracil, and leucovorin (FOLFIRI) in patients with metastatic colorectal cancer who were previously treated with oxaliplatin-containing regimens: A multicenter observational cohort study (TCTG 2nd-BV study). Med Oncol 29: 2842-2848, 2012.

14. Trotti A, Colevas AD, Setser A, et al: CTCAE v3. 0: development of a comprehensive grading system for the adverse effects of cancer treatment. Semin Radiat Oncol 13: 176-181, 2003.

15. Hurwitz H, Fehrenbacher L, Novotny W, et al: Bevacizumab plus irinotecan, fluorouracil, and leucovorin for metastatic colorectal cancer. N Engl J Med 350: 2335-2342, 2004.

16. Giantonio BJ, Catalano PJ, Meropol NJ, et al; Eastern Cooperative Oncology Group Study E3200: Bevacizumab in combination with oxaliplatin, fluorouracil, and leucovorin (FOLFOX4) for previously treated metastatic colorectal cancer: Results from the Eastern Cooperative Oncology Group Study E3200. J Clin Oncol 25: 1539-1544, 2007.

17. Hayashi H, Okamoto I and Nakagawa K: Perirenal hematoma associated with bevacizumab treatment. Invest New Drugs 30: 808-809, 2012

18. Khasraw M, Holodny A, Goldlust SA and DeAngelis LM: Intracranial hemorrhage in patients with cancer treated with bevacizumab: The Memorial Sloan-Kettering experience. Ann Oncol 23: 458-463, 2012.

19. Uysal M, Goksu SS, Coskun HS, Savas B, Ozdogan M and Bozcuk H: Intraarticular hemorrhage due to bevacizumab in a patient with metastatic colorectal cancer: A case report. J Med Case Rep 6: 188, 2012.

20. Tsuchida K, Honjoh Y, Asari M, Osawa E, Numata K, Yoshida T, Osaragi T, Yoneyama K, Kasahara A, Yamamoto Y, et al: A case of rectal hemorrhage during chemotherapy with bevacizumab for local recurrence of rectal cancer. Gan To Kagaku Ryoho 39: 675-677, 2012 (In Japanese). 